\title{
A Study of FMS Part Type Selection Approaches for Short-Term Production Planning
}

\author{
KATHRYN E. STECKE and ILYONG KIM \\ Graduate School of Business Administration, The University of Michigan, Ann Arbor, Michigan
}

\begin{abstract}
This research compares seven approaches from the literature to the selection of part types for simultaneous production over the next time horizon. A flexible approach to the selection of part types and the simultaneous determination of their mix ratios so as to balance aggregate machine workloads is presented. Constraints on tool magazine capacity are considered. Simulation studies are conducted on realistic, detailed models of flexible flow systems (FFSs) configured as pooled machines of equal sizes. The simulated settings are constructed to evaluate the impact of such factors as blocking, transportation, buffer utilizations, and fixture requirements and limitations of various types.

One of the goals of this study is to encourage industry to relax, for those FMS types for which the procedure is appropriate, what is essentially an artificial constraint: that tool changing be isolated in time, to a period between batches. For other types of FMSs, batching may be appropriate.

The results indicate that using the flexible approach enables the system to be more highly utilized. It is also observed that the batching approaches tend to require more fixtures of each type than the flexible approach. The system utilizations for the batching approaches seem to be more sensitive to restrictions on the number of fixtures of each type. Further research needs are also discussed.
\end{abstract}

\section{Introduction}

The short-term production planning function for setting up a flexible manufacturing system (FMS) prior to production should be performed so as to interact well with the operation of the system over an upcoming time horizon. FMS production planning problems are defined as system setup decisions that must be made before an FMS can begin to produce part types. The solutions to these planning problems specify that all cutting tools required for each operation of the selected part types be loaded into the appropriate machines' limited-capacity tool magazines. Then the parts must be scheduled through the system. Production planning should be developed to set up an FMS for subsequent efficient production.

There have been some research studies that address the FMS part type selection problem. This problem is to select a subset of the part types that have been ordered to be produced on an FMS, often with due dates and/or production requirements, for simultaneous machining over some upcoming period of time. This dynamic part type selection problem of short-term planning is not to be confused with the problem of selecting early in the design phase the part types that an FMS will be able to produce. Some algorithms to solve this static, design part type selection problem can be found in Kusiak (1985) and Whitney and Suri (1985), for example. 
During FMS operation, planning for system setup must be performed periodically, for example, when the production requirements are completed for some part types, when the part mix is changed, when a production order needs an expedited entry into the system, or when a machine breaks down. The solution approaches to the part type selection problem can be broadly classified as follows:

A flexible approach to the selection of part types can be implemented as follows. When the production requirements of some part types are finished, space in the tool magazine is freed up. Perhaps some new part types can be introduced into the system for immediate and simultaneous processing, if this input can help the system be more highly utilized (Stecke and Kim (1986a)).

A batching approach partitions the part types into separate sets, called batches, and distinct machining horizons. The selected part types in a particular batch are produced continuously until all the production requirements are completed. Then the system setup time consists of unloading all the cutting tools that are no longer required by the current batch and reloading all new tools to perform the operations of all part types of the next scheduled batch (Whitney and Gaul (1984); Hwang (1986); Rajagopalan (1986)).

To date, research on batching approaches to the selection of part types has been either optimal-seeking or heuristic:

1. Integer programming models. The objective function of Hwang (1986) aims to minimize the number of tool changeovers (i.e., the number of batches). Since this part type selection formulation is intractable, the suggested approach attempts to maximize the number of part types in each batch as a reasonable greedy heuristic. The formulation that minimizes the frequency of tool changeovers seems to postpone as late as possible selecting the part types with the largest number of required cutting tools. In a related study, Rajagopalan (1986) partititions the part types having production requirements into batches. A formulation minimizing total makespan is developed under the assumption of a constant system changeover time. Such an optimization formulation is NP-complete. Hence, several heuristics are suggested.

2. Heuristic methods. Whitney and Gaul (1984) also partition part types into separate batches and distinct machining horizons. The goal is to minimize the number of batches and then to balance workloads within each batch sequentially. The approach is iterative and uses estimated performances indices. The functional values of these indices are not obvious and depend upon the subjective judgment of a user in order to be implemented in a realistic system. Rajagopalan (1986) also suggests heuristic rules using concepts of $m$-dimensional bin packing for selecting part types.

However, no extensive studies to compare these batching approaches on a realistic system have been performed. There are at least three questions concerning the implementation of the existing approaches to the selection of part types in FMSs: (1) For a particular type of FMS, which approach tends to perform better-flexible or batching? (2) Which batching approach performs better? (3) Can a batching approach 
be implemented using a flexible approach? The first issue has been partly dealt with in Stecke and Kim (1986a). The first and second questions are addressed here. The third question is a subject for future research.

A main issue in comparing flexible and batching approaches to FMS part type selection is tool changeover time. Suppose, for example, that a typical FMS consists of eight to ten machine tools, each having a tool magazine capacity of 60 slots. Implementing a batching approach usually requires taking out all cutting tools in all magazines and replacing these with a new set of cutting tools. (This is a total and major tool changeover.) Each individual cutting tool interchange can take about one minute. Changing all tools between batches can take almost a shift or more.

On the other hand, although the flexible approach requires more frequent tool changes, the time required for each tool changeover is greatly reduced. When a part type finishes production, only the affected tools need to be changed. (This can be called a partial or minor tool changeover.) Also, since the remaining part types are still being processed while the affected tools are being changed in a particular tool magazine, the remaining machine tools can continue to cut metal. In addition, if a current operation using a particular cutting tool takes an hour or more to complete, perhaps even some tools in that operating machine can be changed during the cutting. (This is not always possible. Sometimes tools cannot be changed during cutting because of safety reasons or OSHA regulations. It is possible in some systems, though.)

It can be seen that although the total time taken to change all tools may be the same for both the flexible and the batching approaches, the lost, idle time due to tool changeover will be considerably less for the flexible approach.

Another consideration is savings due to labor. Most of the time, there will be a constant number of setup people on the system. The flexible approach to system setup can smooth the use of these people, rather than requiring all of their attention for a large and focused period of time. Cutting tools wear and break randomly, so operators have to be present at all times anyway. The flexible approach can allow for the changing of tools for a part type at the time that the worn and broken tools need to be changed.

These advantages to implementing a more flexible approach may seem clear. However, most researchers as well as FMS users have followed a batching approach, and there are reasons for this. The advantages of using batching rather than the flexible approach are a lower frequency of tool changeovers for batching. In addition, batching may be easier to implement in a real system. The necessary changeover functions are obvious if all tools have to be unloaded. If only a few are removed, the changeover may not be as automatic (although still easy).

In the remainder of this article, the time- and labor-saving advantages of the flexible approach are not considered. Any further benefits are in addition to those just described.

This article analyzes the performances of the flexible approach and various batching approaches over time using a simulation of a realistic flexible flow system (FFS) containing pooled machines of equal sizes. For the batching approaches, the 
FMS production ratio problem is solved to determine the mix ratios of the selected part types to maintain workload balance in the system. The determined production ratios are the relative numbers of parts of the selected part types that are to be in the system together over time. These determined mix ratios of the part types selected by the various approaches are analyzed.

The types of systems that are considered here are those that machine independent part types, each having particular production requirements. There can be more freedom, and hence benefits, in determining the relative production ratios at which a particular set of part types could be machined together. If the requirements of part types are dependent, say, for subsequent assembly, then production ratios, and hence bottlenecks, are prespecified (Stecke (1985)).

The cutting tools required to perform the operations of all part types, and hence tool magazine capacities, are considered here. Duplication of cutting tools shared by several operations is considered, but the possibilities of capacity savings from the overlapping placement of tools in a tool magazine are not modeled here. Due dates are not considered, since some of the proposed batching approaches do not consider due dates.

This paper is organized as follows: In section 2.1, the mathematical programming formulation that selects part types and determines their mix ratios for the flexible approach is presented. Section 2.2 reviews solution procedures that determine aggregate part mix ratios for balancing and unbalancing workloads using the flexible approach. In section 3.1, Hwang's integer formulation for the selection of part types is reviewed. Next, we suggest a different formulation for the same objective of minimizing the number of batches. Also, both Whitney and Gaul's and Rajagopalan's heuristics for the selection of part types are reviewed. Section 3.2 demonstrates how mix ratios of the part types selected using batching approaches are determined. The FMS scenario that is used for the comparisons is described in section 4.1. Section 4.2 provides computational results on finding mix ratios of the part types selected by the various approaches. These are subsequently input into simulations of FFSs. Simulated settings are described in section 5.1, and in section 5.2, computational results of the simulation studies are analyzed. Conclusions and future research needs are given in section 6 .

\section{A part type selection-production ratio model using a flexible approach}

In this section, we show how to select part types and determine their mix ratios for the objectives of balancing or unbalancing workloads using a suggested flexible approach. Notation is provided in table 1.

\subsection{Integer formulation}

Given the types of cutting tools, number of slots occupied by each cutting tool, tool magazine capacity, and aggregate production and processing time requirements of 
Table 1. Notation

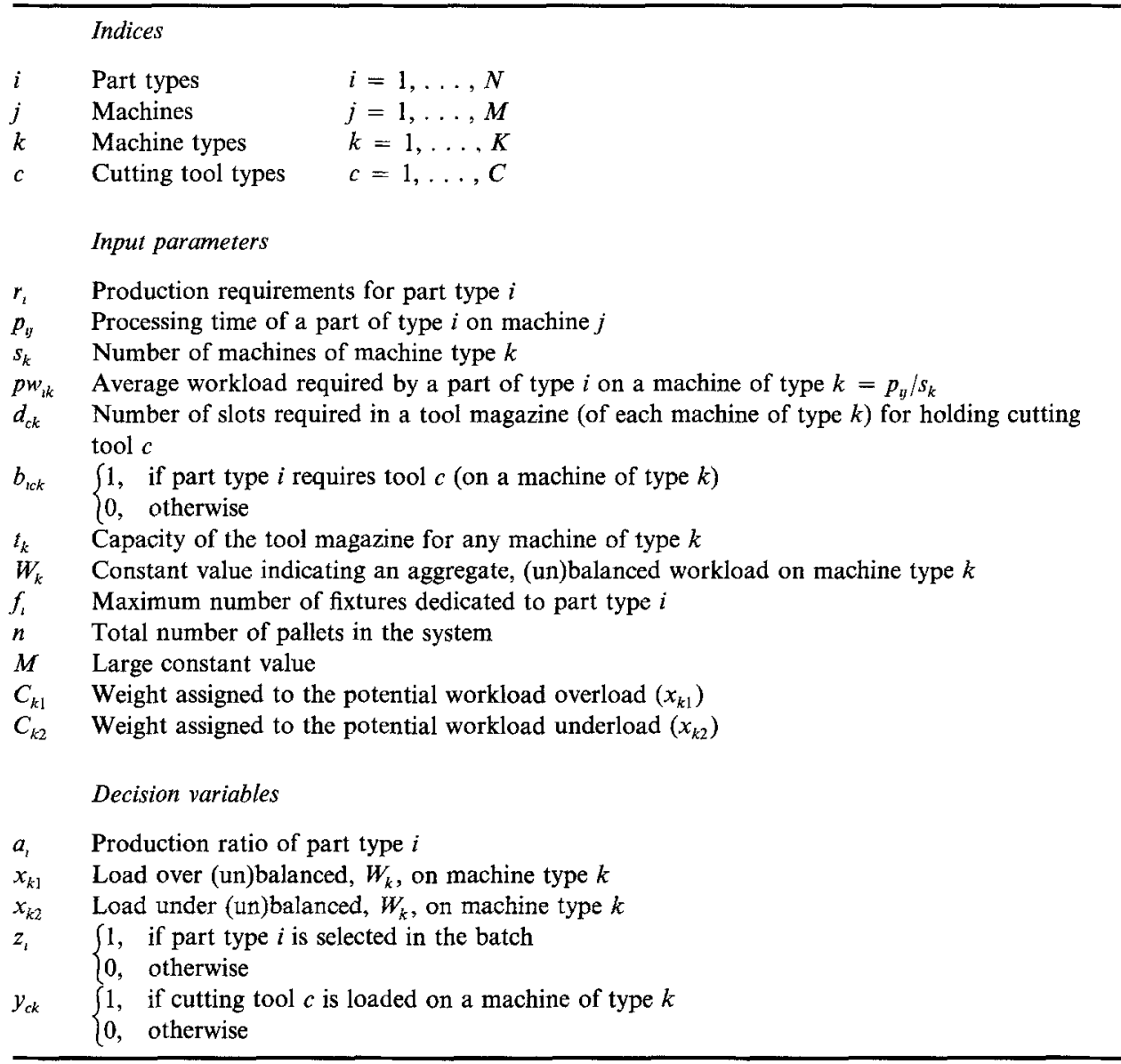

each part type on each machine type, the model to select part types and determine their production ratios is presented as the following integer formulation, model M1:

$$
\begin{aligned}
& \text { Minimize } \quad \sum_{k} C_{k 1} x_{k 1}+\sum_{k} C_{k 2} x_{k 2} \\
& \text { subject to } \quad \sum_{c} d_{c k} y_{c k} \leqslant t_{k}, \quad \text { all } k \\
& b_{c k} a_{i} \leqslant M y_{c k}, \quad \quad \text { all } c \text { and } k \\
& \sum_{1} p w_{t k} a_{1}-x_{k 1}+x_{k 2}=W_{k}, \quad \text { all } k \\
& a_{i} \leqslant f_{i}, \quad \text { all } i
\end{aligned}
$$




$$
\begin{array}{ll}
a_{t} \leqslant 0 \text { and integer, } & \text { all } i \\
y_{c k}=0 \text { or } 1, & \text { all } c \text { and } k \\
x_{k 1}, x_{k 2} \geqslant 0, & \text { all } k
\end{array}
$$

Each operation type can be performed by only one machine type. For simplicity of presentation and notation, this formulation assumes that all machines of each machine type are pooled and that each operation is assigned to all machines of the particular machine type that can perform that operation. The cutting tools for that operation are placed in each machine's tool magazine of the correct machine type. This assumption can be easily relaxed.

The objective function can be changed by weighting the overload and underload on each machine type differently to generate balanced solutions. The over- or underload parameter for each machine type can be weighted arbitrarily to result in different sets of optimal ratios. The optimal production ratios could result in over- or underloading the average workload on some machine types.

Constraint 1 describes the tool magazine capacity on each machine (and hence machine type). The cutting tool assignment is duplicated at each machine of the same machine type. Hence, the tool magazine capacity constraint for a group of machines is identical to the capacity constraint of any single machine in the group. Constraint 2 ensures that if a part type is selected, all of its required cutting tools are loaded in some machine's tool magazine. This constraint also considers tool duplication to ensure that if several operations require some cutting tools in common, those common tools are loaded only once into the appropriate tool magazines. Constraint 3 describes the average workload on each machine type, which can be specified to be unbalanced for systems having pooled machines of unequal sizes (Stecke and Kim (1987a)). The workload parameter, $W_{k}$, can be selected arbitrarily. The relative ratios of workloads on machine types are calculated by using the closed queueing network model, CAN-Q, for groups of pooled machines of unequal sizes (Stecke and Solberg (1985)). We recommend using a small value for $W_{k}$. For example, for the problem sets of section 4 , all $W_{k}$ are 100 , to result in a balanced workload distribution over all machines. This small value for $W_{k}$, results in single-digit production ratios, which may be desirable for operating purposes. Constraint 4 restricts the maximum ratio values (maximum number of parts of each type) to be maintained in the system. This could be caused by a limitation on the number of fixtures of each type.

Part types with near-zero ratio values in the optimal solution are not selected to be in the part mix to be machine together over the upcoming period. The zero production ratios indicate that these part types are not compatible with those selected, with respect to machine utilizations.

Notice that the production requirements for each part type have not yet been considered. These are monitored during system operation. 


\subsection{Solution procedure to select part mix ratios}

In this section, the implementation of the flexible approach using the integer formulation M1 is described. The following algorithm selects the subset of part types to be machined together and determines their aggregate production ratios over some upcoming flexible time period.

\section{PART TYPE SELECTION-PRODUCTION RATIO ALGORITHM}

Step 1. Formulate and solve model M1 for a particular set of parameters $W_{k}, C_{k 1}$, $C_{k 2}$.

Step 2. For those part types with positive production ratio values $\left(a_{t}\right)$ in the optimal solution $\left(a_{l} \geqslant 1\right)$, produce at those ratios until some event occurs, such as the completion of the requirements of some part types.

Step 3. Update the part mix ratios by introducing the following constraints:

$a_{t_{1}} \geqslant 1, \quad$ where $i_{1}=$ part types that have not yet completed their requirements\}

$a_{t_{2}}=0, \quad$ where $i_{2}=$ \{part types that have completed their requirements

$a_{t_{3}} \geqslant 0, \quad$ where $i_{3}=\{$ part types that can be considered for upcoming production

Step 4. If all requirements for all part types are completed, STOP. Otherwise, go to step 1.

The algorithm is reiterated until the requirements of all part types are completed. At Step 2, the part types with near-zero ratio values are not selected to be produced simultaneously over the upcoming time horizon. Step 3 updates the part mix as well as the ratios if the input of one or more new part types makes the machine tools' aggregate workloads more balanced. Otherwise, only the ratios of the same set of part types are updated. The part types that do not complete their requirements continue production over the next horizon without tool changeovers.

The maximum ratio value of each part type should not be larger than the remaining requirements. In this case, additional constraints similar to constraint 2 are introduced. At Step 3, if the total remaining processing times required by some part types having few remaining requirements are small after the completion of requirements of some other part types, it could be more effective to continue production of the remaining part types at updated ratios, rather than considering the introduction of new part types. This saves an unnecessary tool changeover. Then a bound on the total remaining processing time of any one part type, such as half of one shift (four 
hours), is suggested here before any cutting tools are changed. In reality, different bounds could be determined in various situations, either off-line or on-line, by considering the ease and time of tool changeovers, the length of shifts, and the timings of the short tool changeovers already required for wearing or worn cutters.

\section{Batching approaches}

In this section, we present various batching approaches to the selection of part types. The batching approach tries to minimize the frequency of system setup.

\subsection{Part type selection by batching}

Section 3.1.1 reviews Hwang's (1986) formulation for the selection of part types for the objective of maximizing the number of part types in a batch. A different integer formulation for the objective of minimizing the number of batches is suggested in section 3.1.2. The heuristic method developed by Whitney and Gaul is reviewed in section 3.1.3, and section 3.1.4 reviews Rajagopalan's heuristics.

3.1.1. Hwang's integer formulation Given the tools required by each operation of each part type, the number of slots occupied by each tool, and tool magazine capacity of each machine (type), Hwang's formulation to select part types is reviewed as model M2:

$$
\begin{array}{ll}
\text { Maximize } & \sum_{i} z_{t} \\
\text { subject to } & \sum_{c} d_{c} y_{c} \leqslant t \\
& b_{t c} z_{i} \leqslant y_{c}, \quad \text { all } i \text { and } c \\
& z_{i}=0 \text { or } 1, \quad \text { all } i \\
& y_{c}=0 \text { or } 1, \quad \text { all } c
\end{array}
$$

Hwang's model, M2, considers a system of identical machines, all of the same machine type. However, the extension to the case of multiple machine types is immediate if each operation can be performed on only one machine type. The objective function maximizes the number of part types in a batch. Constraint 8 describes the tool magazine capacity of each machine (and hence machine type). Constraint 9 ensures that if a part type is selected, all cutting tools required for all operations of the selected part types are loaded into the tool magazine on each machine of the correct machine type. 
This formulation appears to postpone as late as possible the selection of those part types requiring larger numbers of tool slots, since the objective function maximizes the number of part types in a batch. This leads to a reduced consideration of tool duplication, which can result in an increase in the total number of batches needed to produce all part types. This indicates that model M2 may not always perform well for the original objective of minimizing the frequency of tool changeovers. In order to eliminate this possibility, the following integer formulation is suggested.

3.1.2. Our extention of Hwang's formulation To better satisfy Hwang's chosen criterion, we offer the following alternative objective function in model M3. The coefficient of each part type in the objective function is the number of tool slots required for all operations of that part type. In contrast to Hwang's model, M2, this objective function aims to select early those part types with the most number of required tool slots. Model M3 can reinforce consideration of tool duplication by selecting as early as possible those part types with the largest number of required tool slots (and hence more potential tool overlap can be considered):

$$
\begin{array}{ll}
\text { Maximize } & \sum_{t}\left(\sum_{c} b_{t c} d_{c}\right) z_{\iota} \\
\text { subject to } & (8),(9),(10), \text { and (11) }
\end{array}
$$

An eight-part type example is introduced in table 2 to compare models M2 and M3 for a single machine problem. As noted in table 2 , three batches are required by model M2 to produce all part types, but only two setups for the system are required by model M3.

Model M2 seems to be myopic. Even if the first batch selects more part types, this can lead to a larger than necessary total number of batches to produce all part types. For example, model M2 misses the opportunity that most of the tools required for

Table 2. Comparison of modeis M2 and M3 for part type selection for the objective of minimizing the number of batches

\begin{tabular}{llc}
\hline Part type & $\begin{array}{l}\text { Types of cutting } \\
\text { tools required }\end{array}$ & Results and comparison \\
\hline PT1 & $a$ & Model M2 \\
PT2 & $b$ & Batch 1: PT1, 2, 3, 4, 5, 6 \\
PT3 & $c$ & Batch 2: PT7 \\
PT4 & $d$ & Batch 3: PT8 \\
PT5 & $a, b$ & Model M3 \\
PT6 & $c, d$ & Batch 1: PT2, 3, 4, 5, 6, 8 \\
PT7 & $f, g$ & Batch 2: PT1, 5, 7 \\
PT8 & $b, c, d, e$ & \\
\hline
\end{tabular}

${ }^{a}$ It is assumed that every tool occupies only one slot. Also, the number of slots defining the tool magazine capacity is four. There is one machine. 
part type 8 are shared with the tools required for part types $2,3,4$, and 6 (see table 2).

On the other hand, model 3 tends to consider tool duplication to select part types for a batch. For the example of table 2, part type 8 , having the largest number of required slots, is selected first in the first batch. Therefore, this batch can also include part types $2,3,4$, and 6 , which share the same tools with part type 8 . These models are analyzed more extensively on larger problems and realistic systems in section 4 .

The extension of model M3 to the case of multiple machine types is immediate. In this case, the coefficient of each part type in the objective function is the number of tool slots needed for all operations of that part type required by the largest weighted machine type. The weight for each machine type is the current ratio of tool slots required (for the remaining part types that are to be produced) to its tool magazine capacity.

This objective function is now:

$$
\text { Maximize } \quad \sum_{l}\left(\sum_{c} b_{c c k} d_{c k}\right) z_{l}
$$

Here, the largest weighted machine type, $\vec{k}$, is such that

$$
\bar{k}=\max _{k} \frac{\sum_{t} \sum_{c} b_{x c k} d_{c k}}{t_{\mathrm{k}}}
$$

3.1.3. Whitney and Gaul's method Whitney and Gaul (1984) use a program called BATCHBAL to select part types, which accounts for tool allocation and capacity as well as workload balancing. The main objective of partitioning part types is to minimize the total makespan to produce all part types. Since this part type selection problem is intractable, they use the following two surrogate criteria, which can be conflicting: (1) the number of batches is minimized, and (2) the average utilization of all machines is maximized. The second criterion tends to balance workloads.

BATCHBAL is set up as follows: BATCH attempts to minimize the number of batches. As soon as a batch is determined, BAL investigates whether the batch workloads can be assigned to each machine. If any tool constraints are violated, then BATCH tries again with an alteration, such as a reduction in the tool capacity. The input of BATCHBAL requires the types of cutting tools, number of slots occupied by each cutting tool, tool magazine capacity, and aggregate production and processing time requirements of each part type on each machine type. Cutting tool duplication is considered.

The BATCHBAL program is implemented here in two ways. First, we try to minimize the number of batches using only the BATCH option. Second, we utilize both BATCH and BAL options to balance workloads while minimizing the number of batches. The output of the BATCHBAL program is analyzed in section 4 . 
3.1.4. Rajagopalan's heuristics Rajagopalan (1986) develops six heuristics of two types to solve the part type selection problem. The first type aims to minimize the number of system setups, and the second type tries to balance workloads. For both types, the problem of selecting part types is considered as an $m$-dimensional bin-packing problem, where $m$ is the number of machines. The number of tool slots required by all operations of a part type on a machine is the size of an item. Stecke and Talbot (1985) suggest bin-packing type heuristics for loading FMSs.

In this article, the following two heuristics, shown by Rajagopalan to perform better than his other suggested heuristics, are examined for comparison purposes:

Heuristic I (RHI). That part type is selected (from the remaining part types to be assigned) that requires the maximum total weighted tool slot requirements over all machines. Here, the weight for each machine is the current ratio of tool slot requirements for all operations of all the remaining part types to tool magazine capacity. That is, the weight for machine $j$ is $w_{j}$, where

$$
w_{J}=\frac{\sum_{l} \sum_{c} b_{u j}}{t_{J}} .
$$

Part type $i$ is selected such that

$$
i=\max _{l} \sum_{j} w_{j}\left(\sum_{c} b_{l c} d_{c \jmath}\right)
$$

This selection procedure is iterated until the slots required for all operations of the selected part types satisfy the tool magazine capacity on all machines.

Heuristic II (RHII). That part type is selected (from the remaining part types to be assigned) that minimizes total differences between the ratios of processing time assigned on the different machines to that on the bottleneck machine (in the current batch of part types) and the ratios of processing time remaining on the various machines to that on the bottleneck machine. The processing times of each part type on each machine are weighted by the production requirements. This selection procedure is iterated until the slots required for all operations of the selected part types satisfy the tool magazine capacity on all the machines.

Heuristic I considers the number of tool slots required by each part type to help select part types for an operating objective that tries to minimize the number of major tool changeovers. Heuristic II considers the total production and processing time requirements of all part types on each machine for an operating object of balancing workloads. These are both relevant and useful considerations, for the purpose of efficient machine tool packing. Berrada and Stecke (1986) consider both in the branch-and-bound code for FMS loading. These two heuristics are investigated for flexible flow systems of pooled machines of equal sizes in section 4 . 


\subsection{Production ratio determination}

In this section, we show how to determine production ratios of the part types selected by various batching approaches for the objectives of balancing or unbalancing workloads.

Given aggregate production and processing time requirements of each part type on each machine type, the batching method for determining production ratios is reviewed as the following integer formulation, model M4:

$$
\begin{array}{ll}
\text { Minimize } & \sum_{k} C_{k 1} x_{k 1}+\sum_{k} C_{k 2} x_{k 2} \\
\text { subject to } & a_{i} \geqslant 1 \text { and integer, } \quad \text { all } i
\end{array}
$$

$$
\text { (3), (4), and (7) }
$$

Constraint 12 ensures that the part types selected by batching always have positive integer production ratio values.

The batching approach is implemented as follows. Whenever the requirements for a part type in a particular batch are completed, new production ratios are found for the remaining part types that aim to balance machine workloads as optimally as possible. This attempts to implement batching as favorably as possible.

\section{Part type selection experiments}

In this section, the results of the seven approaches to part type selection are compared using a realistic FMS scenario having pooled machines of equal sizes. Section 4.1 describes the problem sets, and section 4.2 gives the computational results on finding mix ratios of the part types selected by the various approaches. The performances of the seven part types selection approaches are compared in section 5 .

\subsection{Scenarios investigated}

The problem set of table 3 is used to demonstrate the performances of the seven suggested approaches to the selection of part types. There are ten part types with associated production requirements that are ordered to be produced on an FMS having three groups of pooled machines of equal sizes. In particular, there are pooled mills, drills, and vertical turret lathes (VTLs), each group having two identical machines. The processing times (in minutes) and the production requirements for each part type are provided in table 3 for this system of three machine types and six machines. Each part type requires between four and eight different cutting tools on each machine type. Each cutting tool occupies one or three slots. The total number 
Table 3. Processing times and production requirements for ten part types on three machine types with six machines

\begin{tabular}{lllll}
\hline Part type & Mill(2) & Drill(2) & VTL(2) & Production requirements \\
\hline PT1 & $40^{\natural}$ & 50 & 50 & $40^{\mathrm{b}}$ \\
PT2 & 20 & 20 & 40 & 8 \\
PT3 & 40 & 60 & 40 & 30 \\
PT4 & 20 & 30 & 30 & 50 \\
PT5 & 20 & 50 & 20 & 20 \\
PT6 & 50 & 60 & 10 & 14 \\
PT7 & 30 & 30 & 60 & 85 \\
PT8 & 30 & 10 & 20 & 10 \\
PT9 & 50 & 10 & 30 & 25 \\
PT10 & 50 & 30 & 40 & 35 \\
\hline
\end{tabular}

${ }^{\text {a }}$ Procesing times are in minutes.

${ }^{b}$ Production requirements are in number of parts.

of cutting tools required by all ten part types is 14 . The number of tool slots required by all ten part types is 26,28 , and 32 for mills, drills, and VTLs, respectively. (Detailed input data are omitted here for space considerations but are available upon request.) The tool slot capacities of mills, drills, and VTLs are specified as 30, 35, and 20, respectively. Tool overlapping is not considered here, but tool duplication is.

There are two cases of fixture limitations considered here: (1) the case where the number of fixtures of each type is limited to four, and (2) the case where no restrictions on fixtures are required. One reason is to compare the effects of having a limited number of fixtures available. A fixed number of parts (the pallet limitation) of mixed types having nonzero production ratio values is always in the system.

Initially, the number of parts (pallets) in the system is nine for the problem of table 3. The values of parameters $W_{k}, C_{k 1}$, and $C_{k 2}$ of models M1 and M4 are specified as 100,1 , and 1, respectively, as workloads are to be balanced. These balanced workloads maximize expected production rate for systems of pooled machines of equal sizes (Stecke and Morin (1985); Shanthikumar and Stecke (1986)). The integer programs M1, M2, M3, and M4 are run using LINDO on an AMDAHL 5860.

For space considerations, only the one problem of table 3 is presented here in detail. However, the results from this problem are representative of the results typically found using these algorithms.

\subsection{Calculation of the selected part mix ratios}

Mix ratios are determined, both with and without fixture limitations, as follows. First, models M1 and M4 are solved without the fixture limitations. Unless all ratio values are always less than or equal to 4, M1 and M4 are again solved after adding the constraints that restrict the maximum ratio values.

Tables 4-8 provide representative results that are typically found by using the various algorithms. The tables were constructed as follows. For each table, first the 
Table 4. Optimum part mix ratios using Hwang's batching formulation, M2, and model M4

\begin{tabular}{|c|c|c|c|c|c|}
\hline Run & Rule & $\begin{array}{l}\text { Selected } \\
\text { part types }\end{array}$ & $\begin{array}{l}\text { Production } \\
\text { ratios }\end{array}$ & $\begin{array}{l}\text { Objective } \\
\text { function value }\end{array}$ & $\begin{array}{l}\text { CPU time } \\
\text { (seconds) }\end{array}$ \\
\hline 1 & $\begin{array}{l}\text { M2-M4 } \\
\text { M4 }\end{array}$ & $\begin{array}{l}3,5,6 \\
5,6\end{array}$ & $\begin{array}{l}3: 1: 1 \\
1 \cdot 3\end{array}$ & $\begin{array}{r}75 \\
105\end{array}$ & $\begin{array}{l}6.791 \\
0.429\end{array}$ \\
\hline 2 & $\begin{array}{l}\mathrm{M} 2-\mathrm{M} 4^{\mathrm{a}} \\
\mathrm{M} 4^{\mathrm{b}}\end{array}$ & $\begin{array}{l}1,7,9 \\
7,9\end{array}$ & $\begin{array}{l}3: 1: 1 \\
2: 3\end{array}$ & $\begin{array}{l}25 \\
65\end{array}$ & $\begin{array}{l}3.416 \\
0.480\end{array}$ \\
\hline 3 & $\mathrm{M} 2-\mathrm{M} 4^{\mathrm{a}}$ & 2,8 & $4: 4$ & 60 & 2.100 \\
\hline 4 & $\mathrm{M} 2-\mathrm{M} 4^{\mathrm{d}}$ & 4 & 7 & 40 & 1.683 \\
\hline 5 & $\mathrm{M} 2-\mathrm{M} 4^{\mathrm{a}}$ & 10 & 5 & 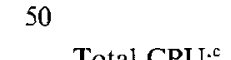 & $\begin{array}{r}1.581 \\
1680\end{array}$ \\
\hline
\end{tabular}

Note: Boldface indicates new part types to be introduced for upcoming run.

${ }^{a}$ New part types scheduled to enter system.

${ }^{6}$ Current ratios updated with no new part types entering.

${ }^{c}$ Total CPU time taken using M2 and M4 only for $a_{t} \leqslant 4$.

corresponding part type selection/production ratio model was run to select part types and their mix. Then a simulation, described in section 5 , was run to see which part type finished its requirements first. With this part type deleted and the remaining part types left on the system, the model was run again to determine new mix ratios and potentially new part types to enter. This iteration continued until all requirements of all part types of table 3 were finished.

Table 4 provides the part mix ratios for the batches selected using both Hwang's model, M2, and the production ratio model, M4. Table 5 provides the part mix ratios for the batches selected using models M3 and M4.

The rules labeled (a) of Tables IV, V, VI, VII, and VIII imply that new part types are scheduled to enter the system. These new part types are noted in boldface. The rules labeled as (b) indicate that the current ratios are updated with no new part type

Table 5. Optimum part mix ratios using the suggested batching formulation M3 and model M4

\begin{tabular}{|c|c|c|c|c|c|}
\hline Run & Rule & $\begin{array}{l}\text { Selected } \\
\text { part types }\end{array}$ & $\begin{array}{l}\text { Production } \\
\text { ratios }\end{array}$ & $\begin{array}{l}\text { Objective } \\
\text { function value }\end{array}$ & $\begin{array}{l}\text { CPU time } \\
\text { (seconds) }\end{array}$ \\
\hline 1 & $\begin{array}{l}\text { M3-M } 4^{\mathrm{a}} \\
\mathrm{M} 4^{\mathrm{b}}\end{array}$ & $\begin{array}{l}3,5,6 \\
5,6\end{array}$ & $\begin{array}{l}3: 1: 1 \\
1: 3\end{array}$ & $\begin{array}{r}75 \\
105\end{array}$ & $\begin{array}{l}6.791 \\
0.429\end{array}$ \\
\hline 2 & $\mathrm{M} 3-\mathbf{M} 4^{\mathrm{a}}$ & $8,9,10$ & $1: 1: 4$ & 75 & 3.841 \\
\hline 3 & $\begin{array}{l}\text { M3-M4 } 4^{\mathrm{a}, \mathrm{c}} \\
\mathrm{M} 3-\mathrm{M} 4^{\mathrm{a}, \mathrm{d}}\end{array}$ & $\begin{array}{l}2,4 \\
2,4\end{array}$ & $\begin{array}{l}2: 4 \\
1: 6\end{array}$ & $\begin{array}{l}60 \\
40\end{array}$ & $\begin{array}{l}2.392 \\
2.289\end{array}$ \\
\hline 4 & M3-M4 & 1,7 & $3: 1$ & 40 & $\begin{array}{r}1.607 \\
15060\end{array}$ \\
\hline
\end{tabular}

Note: Boldface indicates new part types to be introduced for upcoming run.

${ }^{a}$ New part types scheduled to enter system.

${ }^{b}$ Current ratio updated with no new part types entering.

' Second run, with $a, \leqslant 4$.

${ }^{\mathrm{d}}$ Solved without fixture limitations.

${ }^{e}$ Total CPU time taken using M3 and M4 only for $a_{i} \leqslant 4$. 
Table 6. Part mix ratios using BATCHBAL program and model M4

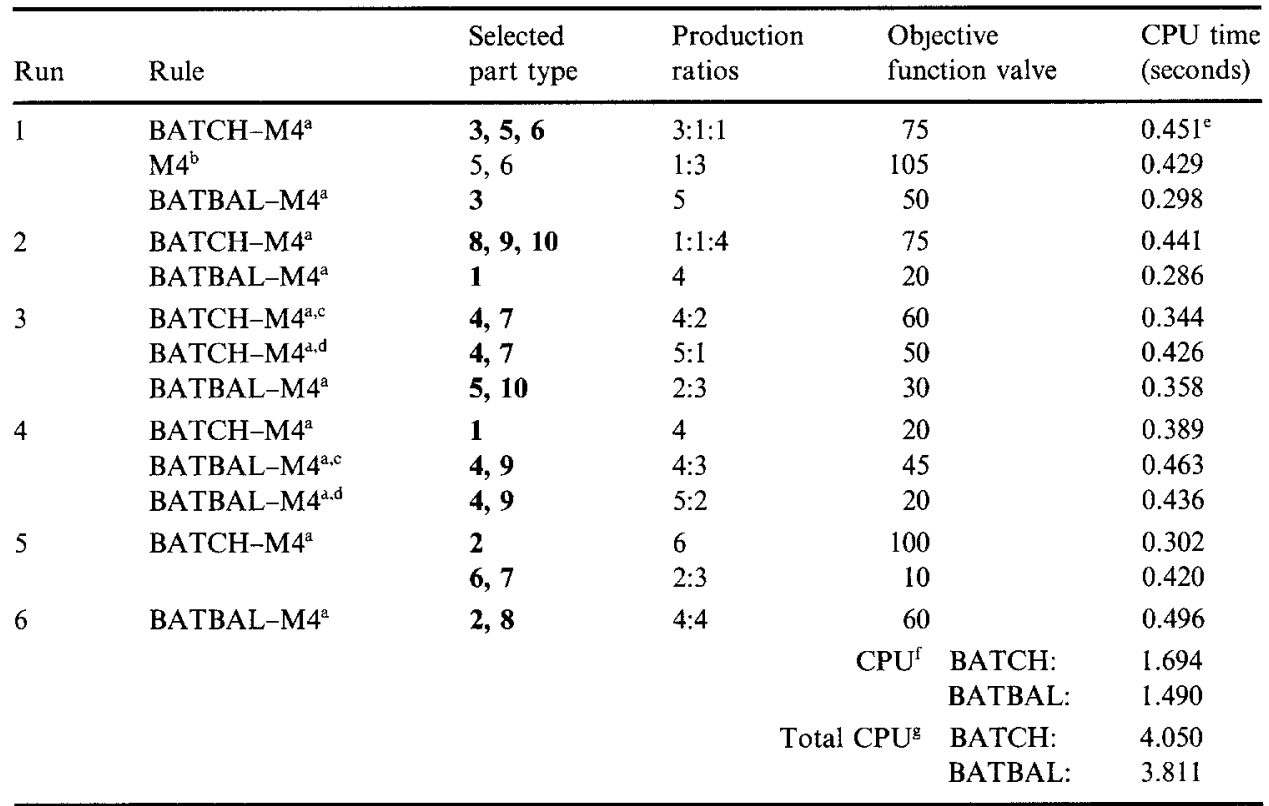

Notes: Boldface indicates new part types to be introduced for upcoming run. BATCH refers to the use of only the BATCH option, and BATBAL (short for BATCHBAL) to the use of both BATCH and BAL options.

a New part types scheduled to enter system.

${ }^{\mathrm{b}}$ Current ratio updated with no new part types entering.

${ }^{\mathrm{c}}$ Second run, with $a_{i} \leqslant 4$.

${ }^{\mathrm{d}}$ No fixture limitations.

${ }^{\circ} \mathrm{CPU}$ time for model M4 using LINDO.

${ }^{\mathrm{f}} \mathrm{CPU}$ time required to partition all part types into batches using the BATCHBAL program and using BATCH alone.

${ }^{\mathrm{g}}$ Total CPU time taken using BATCH (BATCHBAL) and M4 only for $a_{i} \leqslant 4$.

entering. If any part type requires more than four fixtures, M1 and M4 are again solved (second run) after adding the constraint that restricts the maximum ratio values to be no larger than 4 .

Table 6 provides the part mix selected in each batch using BATCH and BAL. Table 6 also demonstrates the use of model M4 to determine production ratios of the selected part types. BATCH tries to minimize the number of batches required to produce all part types. On the other hand, using BATCH and BAL examines whether the workloads for the selected part types in a batch can be balanced on each machine type whenever that batch is determined.

Table 7 provides the part mix ratios for the batches selected using both of Rajagopalan's heuristics, RHI and RHII, and model M4. Table 8 provides the balanced mix ratios of the part types selected using the flexible approach, model M1.

The following observations can be made from tables $4-8$ : 
Table 7. Part mix ratios using Rajagopalan's heuristics and model M4

\begin{tabular}{|c|c|c|c|c|c|c|}
\hline Run & Rule & $\begin{array}{l}\text { Selected } \\
\text { part types }\end{array}$ & $\begin{array}{l}\text { Production } \\
\text { ratios }\end{array}$ & \multicolumn{2}{|c|}{$\begin{array}{l}\text { Objective } \\
\text { function value }\end{array}$} & $\begin{array}{l}\text { CPU time } \\
\text { (seconds) }\end{array}$ \\
\hline 1 & $\begin{array}{l}\text { RHI-M4 } \\
\mathrm{M}^{\mathrm{a}} \\
\text { RHII-M4 } \\
\mathrm{M}^{\mathrm{a}}{ }^{\mathrm{b}}\end{array}$ & $\begin{array}{l}3,5,6 \\
5,6 \\
1,5,6 \\
1,5\end{array}$ & $\begin{array}{l}3: 1: 1 \\
1: 3 \\
2: 1: 2 \\
3: 1\end{array}$ & $\begin{array}{r}75 \\
105 \\
5 \\
45\end{array}$ & & $\begin{array}{l}0.451^{e} \\
0.429 \\
0.631 \\
0.328\end{array}$ \\
\hline 2 & $\begin{array}{l}\text { RHI-M4 }{ }^{\mathrm{a}} \\
\text { RHII-M4 } \\
\text { RHII-M4 } \\
\text { a, }\end{array}$ & $\begin{array}{l}1,10 \\
4,8 \\
4,8\end{array}$ & $\begin{array}{l}3: 1 \\
4: 4 \\
6: 2\end{array}$ & $\begin{array}{l}30 \\
20 \\
20\end{array}$ & & $\begin{array}{l}0.457 \\
0.424 \\
0.519\end{array}$ \\
\hline 3 & $\begin{array}{l}\text { RHI-M4 }{ }^{a} \\
\text { RHII-M4 }\end{array}$ & $\begin{array}{l}2,7 \\
2,7\end{array}$ & $\begin{array}{l}3: 3 \\
3: 3\end{array}$ & \multicolumn{2}{|l|}{$\begin{array}{l}100 \\
100\end{array}$} & $\begin{array}{l}0.349 \\
0.349\end{array}$ \\
\hline 4 & $\begin{array}{l}\text { RHI-M4 } 4^{\mathrm{a}, \mathrm{c}} \\
\text { RHI-M4 } 4^{\mathrm{add}} \\
\text { RHII-M4 }^{\mathrm{a}}\end{array}$ & $\begin{array}{l}4,9 \\
4,9 \\
3,9\end{array}$ & $\begin{array}{l}4: 3 \\
5: 2 \\
3: 2\end{array}$ & \multicolumn{2}{|l|}{$\begin{array}{l}20 \\
20\end{array}$} & $\begin{array}{l}0.474 \\
0.436 \\
0.447\end{array}$ \\
\hline 5 & $\begin{array}{l}\text { RHI-M4 } 4^{\mathrm{a}} \\
\text { RHII-M4 }\end{array}$ & $\begin{array}{l}8 \\
6\end{array}$ & $\begin{array}{l}9 \\
4\end{array}$ & \multicolumn{2}{|l|}{$\begin{array}{l}100 \\
100\end{array}$} & $\begin{array}{l}0.298 \\
0.291\end{array}$ \\
\hline & & & & $\mathrm{CPU}^{\mathrm{f}}$ & $\begin{array}{l}\text { RHI: } \\
\text { RHII: }\end{array}$ & $\begin{array}{l}0.666 \\
0.101\end{array}$ \\
\hline & & & & Total CPU & $\begin{array}{l}\text { RHI: } \\
\text { RHII: }\end{array}$ & $\begin{array}{l}2.524 \\
2.571\end{array}$ \\
\hline
\end{tabular}

Notes: Boldface indicates new part types to be introduced for upcoming run. RHI refers to Rajagopalan's heuristic I, and RHII to his heuristic II.

${ }^{\text {a }}$ New part types scheduled to enter system.

${ }^{b}$ Current ratio updated with no new part types entering.

${ }^{\mathrm{c}}$ Second run, with $a_{t} \leqslant 4$.

${ }^{\mathrm{d}}$ No fixture limitations.

${ }^{\circ} \mathrm{CPU}$ time for model M4 using LINDO.

${ }^{\mathrm{f}} \mathrm{CPU}$ time required to partition all part types into batches using RHI and RHII.

${ }^{8}$ Total CPU time taken using RHI (RHII) and M4 only for $a_{t} \leqslant 4$.

1. Each run dictates a total and significant tool changeover, for the batching approaches. For these, there are four total and major system setups (tool changeovers) for Hwang's model, three total for the suggested model, four total for the BATCH option, five total for both BATCH and BAL options, and four total for both of Rajagopalan's heuristics.

2. There are five minor tool reloadings for the flexible approach.

3. Our suggested model M3 leads to the smallest number of batches required to produce all part types among the seven part type selection approaches, six of which are batching.

4. The objective function values (which measure the extent of system balance) for the batching approaches deteriorate as new ratios for the remaining part types in a particular batch are found (see tables 4-7). This will usually lead to lower system utilization as the system operates. This deterioration occurs because new ratios are found continually without the potentially advantageous introduction of some new part types, which can let the system be more highly balanced and utilized. 
Table 8. Optimum part mix ratios using the flexible approach, MI

\begin{tabular}{|c|c|c|c|c|c|}
\hline Run & Rule & $\begin{array}{l}\text { Selected } \\
\text { part types }\end{array}$ & $\begin{array}{l}\text { Production } \\
\text { ratios }\end{array}$ & $\begin{array}{l}\text { Objective } \\
\text { function value }\end{array}$ & $\begin{array}{l}\text { CPU time } \\
\text { (seconds) }\end{array}$ \\
\hline 1 & $\mathrm{Ml}^{\mathrm{a}}$ & $5,7,8,10$ & $2: 1: 1: 2$ & 0 & 85.466 \\
\hline 2 & $\mathrm{MI}^{\mathrm{a}}$ & $3,7,9,10$ & $2: 1: 1: 1$ & 15 & 16.833 \\
\hline 3 & $\mathrm{MI}^{\mathrm{a}, \mathrm{c}, \mathrm{e}}$ & $\begin{array}{l}4,7,9 \\
4,7,9 \\
4,7\end{array}$ & $\begin{array}{l}3: 1: 2 \\
5: 1: 1 \\
4: 2\end{array}$ & $\begin{array}{l}40 \\
35 \\
60\end{array}$ & $\begin{array}{l}22.204 \\
16.054 \\
31.344\end{array}$ \\
\hline 4 & $\mathrm{Ml}^{\mathrm{d}}$ & 6,7 & $2: 3$ & 10 & 3.288 \\
\hline 5 & $M 1^{a}$ & 1,7 & $4: 1$ & 50 & 3.276 \\
\hline \multirow[t]{2}{*}{6} & $\begin{array}{l}M 1^{\mathrm{a}, \mathrm{c}} \\
\mathrm{M} 1^{\mathrm{a}, \mathrm{d}}\end{array}$ & $\begin{array}{l}2,7 \\
2,7\end{array}$ & $\begin{array}{l}2: 2 \\
7: 2\end{array}$ & $\begin{array}{l}100 \\
100\end{array}$ & $\begin{array}{l}1.371 \\
1.179\end{array}$ \\
\hline & & & & Total $\mathrm{CPU}^{\mathrm{e}}$ & 163.782 \\
\hline
\end{tabular}

Note: Boldface indicates new part types to be introduced for upcoming run.

${ }^{\mathrm{a}}$ New part types scheduled to enter system.

${ }^{\mathrm{b}}$ Current ratio updated with no new part types entering.

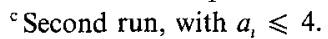

${ }^{\mathrm{d} S}$ Solved without fixture limitations.

${ }^{\mathrm{e}}$ Total CPU time taken using M1 only for $a_{i} \leqslant 4$.

5. The objective function values of the flexible approach also deteriorate with the number of runs. This is because the problem here is static, having fixed orders. In the more typical dynamic situations of orders arriving to an FMS continuously, a better utilization can be anticipated.

6. The CPU time of the flexible approach for the first run is large. This is because tool magazine capacity constraints are considered here concurrently with part type and mix ratio selection for this run. The CPU time decreases with the number of runs. The batching approaches consider tool magazine capacity with part type selection separately from the production ratio determination. The two problems are solved separately.

It should be noted that the objective function values (measuring the extent of system balance) of the determined mix ratios for the flexible approach are better than those for the six batching approaches (except sometimes for the last run). Also, system setup time (tool changeover time) is usually smaller by following a flexible approach. This is because only the few tools affected are changed in a particular tool magazine. Often, the other machine tools can continue to operate.

\section{Simulated settings}

In this section, various approaches to the selection of part types are compared by simulating an FFS of groups of pooled machines of equal sizes. The simulation model is developed in GPSS/H. Schriber (1985) and Schriber and Stecke $(1986 ; 1987)$ have 


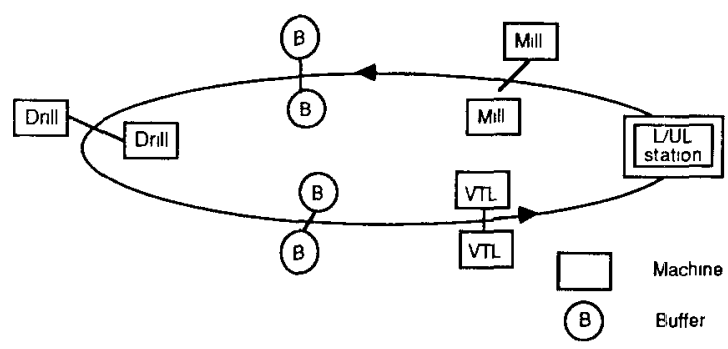

Figure 1. System configuration.

also used a detailed GPSS/H model of an FFS to examine the usefulness of the aggregate production ratios of Stecke (1985) when system realities such as finite numbers of buffers, congestion, blocking, and a finite number of transporters, such as automatic guided vehicles (AGVs) are considered.

\subsection{The simulation model}

The FMS configuration is provided in figure 1 . The following specifications are used for the subsequent experiments:

1. The FFS has unidirectional transportation.

2. Six workstations: two pooled mills, two drills, and two VTLs.

3. Four buffer spaces: two between the mills and drills and two between the drills and lathes.

4. Five load/unload stations.

5. Five carts (wire-guided vehicles).

6. Travel times are one minute between all links, i.e., between L/UL and mill; mill and buffer; buffer and drill; drill and buffer; buffer and VTL; VTL and L/UL (see figure 1).

7. Fixture limitations: (1) the number of fixtures of each type is limited to four from each part type $\left(f_{t} \leqslant 4, i=1, \ldots, 10\right)$, and (2) no restriction on the number of fixtures $\left(f_{i}<\infty, i=1, \ldots, 10\right)$.

8. Nine pallets in the system.

A fixed number of parts of mixed types having nonzero production ratio values is always in the system. For the flexible approach, a current simulation run is usually terminated whenever the production requirements of some part types are finished. When one or more new part types are selected to be input into the system (as specified in table 8), new ratios are found to begin the next run. Otherwise, if no new part type is to enter, the current simulation run continues. However, new optimal production ratios are found for the reduced set of part types. On the other hand, for all batching approaches, a current simulation run continues until all requirements of all part types in one batch are finished. As any part types of a particular batch complete the 
requirements, new production ratios are found for the remaining part types to balance workloads as optimally as possible.

The part input sequence into the FFS here is determined by using a combination of a modified Johnson's algorithm (Campbell, Dudek, and Smith (1970)) and the calculated part mix ratios. See Stecke and Kim (1986b) for a detailed description of this. In particular, for the part types of table 3, the selected part types are always input to the system according to the modified Johnson's algorithm. When a machine and cart become available, a part can be moved. When two or more parts wait for a machine, first-come, first-served (FCFS) is used in the buffer.

\subsection{The simulation results}

Now we present the simulation results to investigate the flexible and batching approaches. Simulations are performed for the problem of table 3 for the two cases of fixture limitations. Tables 9 and 10 provide computational results on the system, machine, and processing utilizations as well as makespan.

Processing (transportation and blocking) utilizations are found for each machine type. These indicate the proportions of total processing (transportation and blocking) times to total makespan. Processing utilization is calculated as the ratio of total actual machining time to total makespan, for each machine type: mill, drill, and VTL.

Table 9. Simulation results after the completion of all production requirements of all ten part types when the number of fixtures of each type is limited to four.

\begin{tabular}{|c|c|c|c|c|c|c|c|}
\hline \multirow[b]{2}{*}{ Comparison } & \multicolumn{6}{|c|}{ Batching approaches } & \multirow{2}{*}{$\frac{\text { Flexible }}{\text { M1 }}$} \\
\hline & M2 & M3 & BATCH & BATBAL & RHI & RHII & \\
\hline Makespan (minutes) & 8,766 & 8,745 & 8,900 & 8,611 & 8,635 & 8,146 & 7,479 \\
\hline Mill utilization & 0.686 & 0.687 & 0.675 & 0.655 & 0.696 & 0.700 & 0.760 \\
\hline Processing utilization & 0.622 & 0.623 & 0.612 & 0.633 & 0.631 & 0.669 & 0.729 \\
\hline Transportation utilization & 0.021 & 0.021 & 0.020 & 0.021 & 0.021 & 0.023 & 0.031 \\
\hline Blocking utilization & 0.043 & 0.043 & 0.043 & 0.001 & 0.044 & 0.008 & 0.000 \\
\hline Drill utilization & 0.703 & 0.681 & 0.672 & 0.691 & 0.696 & 0.737 & 0.820 \\
\hline Processing utilization & 0.641 & 0.643 & 0.632 & 0.653 & 0.651 & 0.690 & 0.752 \\
\hline Transportation utilization & 0.032 & 0.032 & 0.030 & 0.032 & 0.033 & 0.033 & 0.037 \\
\hline Blocking utilization & 0.030 & 0.006 & 0.010 & 0.006 & 0.012 & 0.014 & 0.031 \\
\hline VTL utilization & 0.772 & 0.776 & 0.760 & 0.787 & 0.785 & 0.830 & 0.905 \\
\hline Processing utilization & 0.742 & 0.743 & 0.730 & 0.755 & 0.753 & 0.798 & 0.869 \\
\hline Transportation utilizatıon & 0.030 & 0.033 & 0.030 & 0.032 & 0.032 & 0.032 & 0.036 \\
\hline Blocking utilization & 0.000 & 0.000 & 0.000 & 0.000 & 0.000 & 0.000 & 0.000 \\
\hline System utilization & 0.668 & 0.670 & 0.658 & 0.680 & 0.678 & 0.719 & 0.783 \\
\hline Average buffer utilization & 0.183 & 0.160 & 0.186 & 0.065 & 0.148 & 0.188 & 0.308 \\
\hline Cart utilizatıon & 0.046 & 0.046 & 0.044 & 0.046 & 0.047 & 0.050 & 0.062 \\
\hline Number of dedicated fixtures & 40 & 39 & 40 & 40 & 40 & 40 & 38 \\
\hline
\end{tabular}

Note: Boldface indicates best performance. 
Table 10. Simulation results after the completion of all production requirements of all ten part types when there are no limitations on the number of fixtures of each type

\begin{tabular}{llllllll}
\hline & \multicolumn{3}{l}{ Batching } & & & & Flexible \\
\cline { 2 - 8 } Comparison & M2 & M3 & BATCH & BATBAL & RHI & RHII & M1 \\
\hline Makespan (minutess) & 8,217 & 8,395 & 8.450 & 7,764 & 8,107 & 7,815 & $\mathbf{7 3 8 3}$ \\
Mill utilization & 0788 & 0.808 & $\mathbf{0 . 8 2 7}$ & 0.813 & 0.780 & 0.751 & 0.790 \\
$\quad$ Processing utilization & 0.663 & 0.649 & 0.645 & 0.702 & 0.672 & 0.698 & $\mathbf{0 . 7 3 8}$ \\
$\quad$ Transportation utilization & 0.024 & 0.021 & 0.033 & 0.029 & 0.025 & 0.026 & 0.038 \\
$\quad$ Blocking utilization & 0.101 & 0.138 & 0.149 & 0.082 & 0.083 & 0.027 & 0.014 \\
Dr1ll utilization & 0.893 & 0.842 & 0.834 & 0.891 & 0.895 & 0.913 & $\mathbf{0 . 8 8 1}$ \\
Processing utilization & 0.684 & 0.670 & 0.665 & 0.724 & 0.693 & 0.719 & $\mathbf{0 . 7 6 1}$ \\
$\quad$ Transportation utilization & 0.027 & 0.025 & 0.028 & 0.033 & 0.029 & 0.028 & 0.036 \\
$\quad$ Blocking utilization & 0.182 & 0.147 & 0.141 & 0.134 & 0.173 & 0.166 & 0.084 \\
VTL utilization & 0822 & 0.808 & 0.805 & 0.875 & 0.834 & 0.863 & $\mathbf{0 . 9 1 9}$ \\
$\quad$ Processing utilization & 0.791 & 0.774 & 0.769 & 0.837 & 0.802 & 0.832 & $\mathbf{0 . 8 8 1}$ \\
$\quad$ Transportation utilization & 0.031 & 0.029 & 0.031 & 0.038 & 0.032 & 0.031 & 0.038 \\
$\quad$ Blocking utilization & 0.000 & 0.005 & 0.005 & 0.000 & 0.000 & 0.000 & 0.000 \\
System utilization & 0.713 & 0.698 & 0.693 & 0.754 & 0.722 & 0.750 & $\mathbf{0 . 7 9 3}$ \\
Average buffer utilization & 0.568 & 0.554 & 0.507 & 0.498 & 0.599 & 0.587 & 0.526 \\
Cart utilization & 0.050 & 0.047 & 0.054 & 0.059 & 0.053 & 0.055 & 0.068 \\
Number of dedicated fixtures & 71 & 65 & 72 & 71 & 69 & 63 & 54 \\
\hline
\end{tabular}

Note: Boldface indicates best performance.

Machine utilization is expressed as the sum of processing, transportation, and blocking utilizations, for each machine type. System utilization is an average of the processing utilizations of the three machine types and is a measure of overall system usage. The difference between machine and processing utilizations provides the average amount of time spent in transportation and blocked.

The simulation results demonstrate how much the system and processing utilizations are improved by using the flexible approach. The boldface values in tables 9 and 10 note the best performances among the seven approaches. The following observations can be made:

1. The flexible approach results in higher system utilization than batching. This is consistent with the decrease in makespan for the flexible approach.

2. When there are no required fixture limitations, the flexible approach requires many fewer dedicated fixtures than batching (see table 10). This is because when all requirements of the selected part types in a particular batch except for one part type are completed, batching has only the remnants of that single part type having remaining requirements to process. These few remaining requirements would require additional fixtures (if they were available) for that part type to be finished (or alternatively, longer makespan).

3. The system utilizations for various batching approaches are much better when there are no fixture limitations than when there is the limit of four fixtures of each 
type. This indicates that utilization when batching seems to be sensitive to fixture limitations.

4. The amount of blocking for all three machine types is larger for the batching approaches than for the flexible approach when there are no required fixture limitations.

5. Even though the BATCH option is better than BATCH and BAL options simultaneously in terms of the number of tool changeovers (see table 6), the use of BATCH and BAL options used together leads to better machine utilizations. This is because BATCH and BAL options attempt to balance workloads while partitioning the part types into batches.

5. Among the six batching approaches, Rajagopalan's heuristic II and BATCH and BAL options used together lead to higher system utilization. This seems to be because these batching approaches to the selection of part types consider the processing time requirements of each part type to balance workloads on machines. On the other hand, the remaining batching approaches that select part types only try to minimize the number of tool changeovers.

Therefore, it can be noted that the flexible approach increases overall system utilization and also decreases system makespan, at least in the situations examined to date. These positive results are in addition to the savings in tool changeover times. Further studies are required, however.

\section{Conclusions}

This article investigates and compares the performances of seven different approaches to the selection of part types for simultaneous production for a flexible flow system having groups of pooled machines of equal sizes. It also suggests a global procedure for solving part type selection and production ratio determination problems together and over time using a flexible approach to implement the short-term production planning function while balancing machine workloads.

Simulation results are performed to compare the flexible and various batching approaches. They demonstrate that the use of the flexible approach tends to make the system more highly utilized. It is observed that the batching approaches require more fixtures of each type than the flexible approach. The system utilizations for the batching approaches seem to be sensitive to restrictions on the number of fixtures of each type. It can be concluded that this fiexible approach to part type selection can lead to better system utilization and can cope more easily with dynamic situations during operation, for the types of FMSs considered so far.

FMS users should consider following such a more flexible approach to selecting part types dynamically in systems for which the approach may help system performance. For example, if the production requirements for some part types are independent, mix ratios proportional to the requirements will usually hurt system performance. 
Among the six batching approaches, the second heuristic of Rajagopalan (RHII) and using the BATCH and BAL options together in the BATCHBAL program (which considers the processing time requirements of each part type to balance workloads on machines) lead to better system utilization than the other batching approaches. The suggested extension of Hwang's formulation leads to the smallest number of batches to produce part types among the seven part type selection approaches.

There are further research needs concerning FMS part type selection problems. For example, the results presented here are only for one general type of problem. This problem is representative of the performances of the various procedures, but additional studies should be performed to determine which methods are appropriate under which conditions and for what types of systems. Both performance and computational times of the algorithms may be important, depending on the frequency of application.

Also, similar studies should be performed in a more dynamic situation, when there are often changes in production orders or random machine failures. Implementation of some of the present results in more general situations is being developed. Also, the appropriateness of implementing a batching objective using a flexible approach is being further examined.

Similar studies of the various FMS part type selection approaches should be performed when due dates are considered. For most of the approaches compared here, due dates have not yet been incorporated into the algorithms, yet this is an important application. For example, some systems produce the same parts all the time to maintain certain buffer levels, and thus due dates may be less important. Customer due dates are important in other FMSs, however. In fact, considering due dates is more important during part type selection than during scheduling. Those part type selection approaches that do not yet account for due dates should incorporate these considerations into the algorithms.

\section{Acknowledgements}

We wish to thank Mr. Sampath Rajagopalan of Carnegie-Mellon University for kindly sending us a tape of his eight-part type selection heuristics for this testing. We would also like to thank the referees and associate editor for their careful reading and insightful comments. Kathryn E. Stecke would like to acknowledge partial support for this work from NSF Grant No. ECS 8406407 as well as from a grant from the Graduate School of Business Administration of The University of Michigan.

\section{References}

Berrada. M. and Stecke, K. E., "A Branch-and-Bound Approach for Machine Load Balancing in Flexible Manufacturing Systems," Management Sctence 32, pp. 1316-1335 (October 1986). 
Campbell, H. G., Dudek, R. A. and Smith, M. L., "A Heuristic Algorithm for the $n$ Job, $m$ Machine Sequencing Problem." Management Science 16, pp. 630-637 (June 1970).

Hwang. S., "Part Selection Problems in Flexible Manufacturing Systems Planning Stage," in Proceedings of the Second ORSA/TIMS Conference on Flexible Manufacturing Systems (Ann Arbor, MI), K E Stecke and R. Suri (eds.) Amsterdam: Elsevier, (1986), pp. 297-309

Kusiak, A., "The Part Families Problem in Flexible Manufacturng Systems," Annals of Operations Research 3: pp. 279-300 (1985).

Rajagopalan, S. "Formulation and Heuristic Solution for Parts Grouping and Tool Loading in Flexıble Manufacturing Systems," in Proceedings of the Second ORSA/TIMS Conference on Flexible Manufacturing Systems (Ann Arbor, MI), K. E. Stecke and R. Suri (eds.) Amsterdam: Elsevier (1986), pp. 311-320.

Schrage, L. E., Linear Programming Models with LINDO. Scientific Press, Palo Alto, CA (1981).

Schriber, T. J., "A GPSS/H Model for a Hypothetical Flexible Manufacturing System," Annals of Operations Research 3, pp. 171-188 (1985).

Schriber, T. J. and Stecke, K. E., " Machine Utilizations and Production Rates Achieved by Using Balanced Aggregate FMS Production Ratios in a Simulated Setting," in Proceedings of the Second ORSA/TIMS Conference on Flexible Manufacturing Systems (Ann Arbor, MI), K. E. Stecke and R. Suri (eds.) Amsterdam: Elsevier (1986) pp. 405-416.

- "Using Mathematical Programming and Simulation to Study FMS Machine Utilizations," in Proceedings of the 1987 Winter Simulation Conference, A. Thesen, H. Grant, and W. D. Kelton, (eds.) San Diego, CA: Society for Computer Simulation (1987).

Shanthikumar, J. G. and K. E. Stecke. "Reducing Work-in-Process Inventory in Certain Classes of Flexible Manufacturıng Systems." European Journal of Operational Research 26, pp. 266-271 (August 1986).

Stecke, K. E. "Procedures to Determine Both Appropriate Production Ratios and Minimum Inventory Requirements to Maintain These Ratios in Flexible Manufacturing Systems." Working Paper 448. Graduate School of Business Admınıstration, The University of Michigan, Ann Arbor, MI 48109 (1985)

Stecke, K. E., and I. Kim. "A Flexible Approach to Implementing the Short-Term FMS Planning Function," in Proceedings of the Second ORSA/TIMS Conference on Flexible Manufacturing Systems (Ann Arbor. MI), K. E. Stecke and R. Suri (eds.) Amsterdam: Elsevier, (1986a) pp. 283-295.

. "Decision Aids for FMS Part Type Selection Using Aggregate Production Ratios to Study Pooled Machines of Unequal Sizes." Working Paper 478. Graduate School of Business Administration, University of Michigan, Ann Arbor, MI 48109 (1986b).

"A Study of Unbalancing and Balancing for Systems of Pooled Machines of Unequal Sizes," in Proceedings of the IEEE Robotics and Automation Conference (Raleigh, NC), (1987a).

. "Comparison of Various Approaches to the Part Type Selection Problem in Flexible Manufacturing Systems," in Proceedings of the Ninth International Conference on Production Research (Cincinnati, OH), A. Mital, (ed.) (1987b).

Stecke. K. E. and T. L. Morin. "The Optimality of Balancing Workloads in Certain Types of Flexible Manufacturing Systems," European Journal of Operational Reserach 20, pp. 68-82 (April 1985).

Stecke, K. E. and J. J. Solberg, "The Optimality of Unbalancing Both Workloads and Machine Group Sizes in Closed Queueing Networks of Multiserver Queues," Operattons Research 33, pp. 882-910 (July-August 1985).

Stecke, K. E. and F. B. Talbot, "Heuristics for Loading Flexible Manufacturing Systems," in Flexible Manufacturing Recent Developments in FMS, Robotics, CAD/CAM, CIM, A. Raouf and S. I. Ahmad, (eds.) Amsterdam: Elsevier (1985) pp. 73-84.

Whitney, C. K. and T. S. Gaul. "Sequential Decision Procedures for Batching and Balancing in FMSs," in Proceedings of the First ORSA/TIMS Conference on Flexible Manufacturng Systems (Ann Arbor, MI), K. E. Stecke and R. Suri (eds.) Amsterdam: Elsevier (1984) pp. 243-248.

Whitney, C. K. and R. Surı. "Algorithms for Part and Machine Selection in Flexible Manufacturing Systems," Annals of Operations Research 3: pp. 239-261 (1985). 